\title{
Radiosensitizing effect of c-Met kinase inhibitor BPI-9016M in esophageal squamous cell carcinoma cells in vitro and in vivo
}

\author{
Chenxue Jiang ${ }^{1,2}$, Shuiyun $\mathrm{Han}^{3}$, Xiaojiang Sun ${ }^{3}$, Yaping Xu ${ }^{2,3}$, Jianguo Feng ${ }^{4}$, Jinbiao Shang ${ }^{1,5}$ \\ ${ }^{1}$ First Clinical Medical School, Wenzhou Medical University, Wenzhou, China; ${ }^{2}$ Department of Radiation Oncology, Shanghai Pulmonary Hospital, \\ Tongji University School of Medicine, Shanghai, China; ${ }^{3}$ Department of Radiation Oncology, Cancer Hospital of University of Chinese Academy of \\ Sciences (Zhejiang Cancer Hospital), Hangzhou, China; ${ }^{4}$ Cancer Research Institute, Zhejiang Cancer Hospital, Hangzhou, China; ${ }^{5}$ Key Laboratory \\ of Head \& Neck Cancer Translational Research of Zhejiang Province, Hangzhou, China \\ Contributions: (I) Conception and design: J Shang, J Feng, Y Xu; (II) Administrative support: J Shang, J Feng; (III) Provision of study materials or \\ patients: Y Xu, J Feng; (IV) Collection and assembly of data: C Jiang, S Han, X Sun; (V) Data analysis and interpretation: C Jiang, S Han, J Feng; (VI) \\ Manuscript writing: All authors; (VII) Final approval of manuscript: All authors. \\ Correspondence to: Prof. Jinbiao Shang. First Clinical Medical School, Wenzhou Medical University, Wenzhou, China; Key Laboratory of Head \& \\ Neck Cancer Translational Research of Zhejiang Province, Hangzhou, China. Email: 13605807802@163.com.
}

\begin{abstract}
Background: c-Met is the receptor of hepatocyte growth factor (HGF) which plays a key role in inhibiting apoptosis. BPI-9016M is a small-molecule c-Met inhibitor that can promote apoptosis and enhance the cytotoxicity of various DNA-damaging agents. Here, we evaluated the radiosensitizing potential of BPI9016M in Eca109 human esophageal squamous cell carcinoma (ESCC) cells in vitro and in vivo.

Methods: Cell Counting Kit-8 (CCK-8) assay was used to measure cell viability. Clonogenic survival assay and a murine tumor xenograft in male nude mice were used to evaluate the radiosensitizing effect of BPI9016M. Apoptosis was determined by the terminal deoxynucleotidyl transferase dUTP nick end labeling (TUNEL) staining and flow cytometry experiment. Apoptosis-related proteins were detected by western blot. By evaluating the activation of the ATR-Chk1/ATM-Chk2 pathway to detect radiation-induced DNA double-strand break and homologous recombination repair. BPI-9016M induced a radiosensitizing effect in Eca109 cells and reduced the survival rate of clone formation in vitro.

Results: The combination of BPI-9016M with irradiation (IR) significantly delayed the growth of ESCC tumor xenografts than treatment alone $(\mathrm{P}<0.05)$. The radiosensitizing effects of BPI-9016M were due to increased apoptosis, such as the up-regulation of cleaved-caspase 3 and 9, down-regulation of mutant P53 and Bcl-2, the decreased of phosphorylation of ATR and ATM, and the inhibition of $\gamma-\mathrm{H} 2 \mathrm{AX}$ accumulation in vitro and in vivo.
\end{abstract}

Conclusions: These findings indicated that BPI-9016M exerts a radiosensitizing effect and enhances apoptosis by inhibiting homologous recombination DNA repair in irradiated ESCC cells.

Keywords: c-Met; esophageal squamous cell carcinoma (ESCC); radiosensitivity; BPI-9016M

Submitted Nov 24, 2021. Accepted for publication Dec 23, 2021.

doi: $10.21037 / \mathrm{atm}-21-6586$

View this article at: https://dx.doi.org/10.21037/atm-21-6586

\section{Introduction}

Esophageal squamous cell carcinoma (ESCC) is the dominant histological type of esophageal cancer in Eastern Europe and Asia, while 95\% esophageal cancers in China are ESCC (1). Chemoradiotherapy and surgical resection are the standard therapy for locally advanced ESCC; however, the majority of patients with ESCC experience regional or distant treatment failure and have a 5 -year survival rate of only $20-25 \%(2,3)$.

Owing to increased knowledge of the molecular mechanisms that lead to esophageal cancer, a variety of molecular targeted agents have been developed. 
Numerous randomized trials have investigated the efficacy of multimodality therapy combined with molecular targeted agents, with the aim of improving safety without compromising effectiveness, reducing local recurrence, and prolonging survival (4-7).

The hepatocyte growth factor (HGF) receptor (MET, or c-Met) is a potential therapeutic target in a number of cancers, including esophageal cancer. The membrane-based receptor tyrosine kinase c-Met mediate cell migration, invasion and angiogenesis via binds to its ligand HGF. c-Met is overexpressed in 34-54\% of cases of esophageal adenocarcinoma and is significantly associated with survival $(8,9)$. While c-Met is only overexpressed in approximately $7 \%$ of cases of ESCC and not associated with prognosis in patients from Western countries, overexpression of c-Met has been reported in up to $34 \%$ of cases of ESCC and is associated with poor prognosis in patients from China $(10,11)$.

A number of agents have been developed to target c-Met, including BPI-9016M, a novel small-molecule inhibitor that simultaneously target both c-Met and AXL tyrosine kinases (11-14). Basic and clinical studies of these targets have been conducted in esophageal cancer, with most focusing on esophageal adenocarcinoma, which may result the fact esophageal cancer is mainly adenocarcinoma in western countries (15-18). A recent trial reported that foretinib could enhance radiosensitivity in ESCC by phosphorylating c-Met (11). However, BPI-9016M was only reported on the correlation between survival and prognosis in advanced solid tumor (13) and few studies have investigated the mechanism of action of BPI-9016M on c-Met signaling in ESCC.

We thus investigated the radiosensitizing effect of the c-Met inhibitor BPI-9016M in human ESCC Eca109 cells in vitro and in vivo, and explored the associated mechanisms.

We present the following article in accordance with the ARRIVE reporting checklist (available at https://dx.doi. org/10.21037/atm-21-6586).

\section{Methods}

\section{Drugs, cell culture, and irradiation (IR)}

BPI-9016M was prepared as a $10 \mathrm{mg} / \mathrm{mL}$ stock solution in dimethyl sulfoxide (DMSO) and stored at $-20{ }^{\circ} \mathrm{C}$. Eca109 cells were obtained from the Laboratory of Zhejiang Cancer Institute. Cells were cultured in RPMI-1640 medium supplemented with $10 \%$ fetal bovine serum, $100 \mu \mathrm{g} / \mathrm{mL}$ of streptomycin, and $100 \mathrm{of} \mu \mathrm{g} / \mathrm{mL}$ penicillin. Eca109 cells were kept at $37{ }^{\circ} \mathrm{C}$ in a humid environment containing $5 \% \mathrm{CO}_{2}$. X-ray radiation was delivered by a $6-\mathrm{MV}$ linear accelerator (Elekta, Stockholm, Sweden). The dose rate was $250 \mathrm{cGy} / \mathrm{min}$ and the source-to-target distance was $100 \mathrm{~cm}$.

\section{Cell proliferation assay}

Cell viability was assessed using Cell Counting Kit-8 (CCK-8) assay. Eca109 cells were seeded in 96-well plates (4,000 cells/well) 24 hours before drug treatment. BPI$9016 \mathrm{M}$ was added at various concentrations to replicate wells. After 48 hours, $10 \mu \mathrm{L}$ of cell CCK- 8 reagent (Dojindo Laboratories, Kumamoto, Japan) was added and incubated at $37^{\circ} \mathrm{C}$ for 2 hours, with the absorbance being measured at $450 \mathrm{~nm}$ using a spectrophotometer (Thermo Fisher Scientific, Waltham, MA, USA). Each experiment was repeated 3 times.

\section{Determination of radiosensitivity in vitro}

Growing cells were treated with $3.0 \mu \mathrm{g} / \mathrm{mL}$ BPI-9016M for 24 hours; irradiated with $0,2,4,6,8,10$ or 12 Gy using $6 \mathrm{MV}$ X-rays; and then seeded into 96-well plates (Costar; Corning Life Sciences, Glendale, AZ, USA) at various cell numbers depending on the dose of IR. After 7 days of culture in complete medium, the cells were stained with crystal violet and survival colonies containing more than 50 cells were directly scored by an inverted microscope.

Cell survival was plotted using the IR dose as the abscissa axis and the surviving fraction (SF) as the vertical axis. The average lethal dosage of cell (D0) and the quasi-field dosage (Dq), which indicates the ability of cells to repair sublethal injury, and $\mathrm{N}$ were calculated. SF was calculated as follows:

$$
S F=1-\left[1-\exp ^{-\left(\frac{D}{D 0}\right)}\right]^{N} ; D q=\frac{\operatorname{In} N}{(1 / D 0)}
$$

The sensitization enhancement ratio (SER) was calculated as follows: SER = control group D0/treatment group D0.

\section{Cell cycle distribution}

Cells were treated with 6 Gy IR, BPI-9016M $(3.0 \mu \mathrm{g} / \mathrm{mL}$, 48 hours) or both, with DMSO being used as a control. Cells were harvested, fixed with $70 \%$ ethanol, stored overnight at $-20{ }^{\circ} \mathrm{C}$, centrifuged at $300 \mathrm{~g}$, washed twice with phosphatebuffered saline (PBS), and then labeled with $50 \mathrm{mg} / \mathrm{mL}$ 
propidium iodide (PI), and after incubation for 30 minutes in the dark, flow cytometry was used for analysis (CellQuest version 3.1; Beckman Coulter, Inc., Brea, CA, USA).

\section{Apoptosis assays}

Apoptosis was assessed using Annexin V/PI double staining. Cells were treated with 6 Gy IR, BPI-9016M $(3.0 \mu \mathrm{g} / \mathrm{mL}$, 48 hours) or both, with DMSO being used as a control. Cells were stained with Annexin V-FITC and PI, and the apoptotic fraction was mearused by flow cytometry (BD FACSCalibur; BD Biosciences, Franklin Lakes, NJ, USA).

\section{Western blot analysis}

Total proteins were extracted by radioimmunoprecipitation assay (RIPA) buffer containing phenylmethanesulfonyl fluoride (PMSF) and quantified by a bicinchoninic acid (BCA) protein assay kit (Lianke, Hangzhou, China). Proteins $(40 \mu \mathrm{g})$ were isolated by $10 \%$ SDS PAGE electrophoresis, transferred to polyvinylidene difluoride (PVDF) membranes, blocked and incubated with primary antibody, incubated with horseradish peroxidase (HRP)conjugated secondary antibodies $(1: 2,000)$ and detected by enhanced chemiluminescence (ECL).

\section{Tumor xenograft model}

A protocol was prepared before the study without registration. Animal treatments were performed according to institution-approved protocols. The animal experiments were approved by the Institutional Animal Care and Use Committee of Zhejiang Cancer Research Institute (Hangzhou, Zhejiang, China) (No. 2021-12005), in compliance with the National Institutes of Health Guide for the Care and Use of Laboratory Animals (19). Four-week-old male nude mice purchased from Hangzhou Hangsi Biological Technology Company were subcutaneously injected with $1 \times 10^{6}$ Eca109 cells in $0.1 \mathrm{~mL}$ PBS into the right leg. Nude mice were randomly allocated to a control group, BPI-9016M group, IR group, or IR plus BPI-9016M group, with 6 mice per group. After the nude mice were numbered by one researcher, another researcher took the numbers from the sealed, opaque envelope and randomly divided them into four groups. The sample size was based on published literature on radiation sensitization $(20,21)$. Daily administration of $37.5 \mathrm{mg} / \mathrm{kg}$ of BPI-9016M by gavage was started on day 11 (when the tumors exceeded $5 \mathrm{~mm}$ in any direction; dosage was based on a clinical trial NCT02478866). The nude mice of the IR and IR plus BPI9016M group received 8 Gy of IR on day 19 after drug treatment had been completed. All nude mice were raised in a specific pathogen-free environment.

Xenograft tumor IR was performed using a small animal radiation research platform (SARRP; Gulmay Medical, Sugar Hill, GA, USA) at $50 \mathrm{kVp}$ and $0.8 \mathrm{~mA}$ using $10 \mathrm{~mm}$ of $\mathrm{Cu}$ filtration in the imaging mode and at $200 \mathrm{kVp}$ and $13 \mathrm{~mA}$ using $10 \mathrm{~mm}$ of $\mathrm{Cu}$ filtration in the therapy mode. Mice were anesthetized with $1 \%$ pentobarbital sodium $(100 \mathrm{mg} / \mathrm{kg})$ and immobilized on the 4-axis robotic positioner. Xenograft tumors were irradiated using a $20 \times 20 \mathrm{~mm}$ collimator using the image-guided micro-IR (IGMI) technique; dose exposure calibration and isocenter selection for targeted radiotherapy were performed using Xstrahl MuriSlice Software (Suwanee, GA, USA). The dose rate was $3.69 \mathrm{~Gy} /$ minute.

The body weight of these mice and tumor diameter were measured every 3 days, and tumor volume was determined according to the formula: (width ${ }^{2} \times$ length) divided by the 2 . Growth delay (GD) was determined as the time for treated tumors to double in volume minus the time for control tumors to double in volume. The enhancement factor (EF) was calculated as follows: $\mathrm{EF}=\left(\mathrm{GD}_{\mathrm{IR}}+\mathrm{BPI}-9016 \mathrm{M}-\mathrm{GD}_{\mathrm{BPI}-9016 \mathrm{M}}\right)$ divided by the GDIR.

The data collector did not participate in the abovementioned experimental operation. Mice were killed on day 28 or if the tumor volume of any nude mice exceeded $2 \mathrm{~cm}^{3}$. Total proteins were extracted from tumor samples using RIPA/PMSF buffer and quantified using a BCA protein assay kit. Tumor specimens were fixed in $10 \%$ formalin. The paraffin-embedded were sectioned $5 \mu \mathrm{m}$ thick for immunohistochemical (IHC) or terminal deoxynucleotidyl transferase dUTP nick end labeling (TUNEL) assay.

\section{Immunobistochemistry}

An esophageal carcinoma tissue chip which contain samples of the 24 xenograft tumor tissues from the 6 nude mice per group was created. IHC was performed according to a method described previously (22). Light microscopy was used to analyze the chip. The labeling index was equal to the ratio of the number of cleaved caspase 3 -positive cells to the total number of nuclei (4 fields per 1,000 tumor cells). 

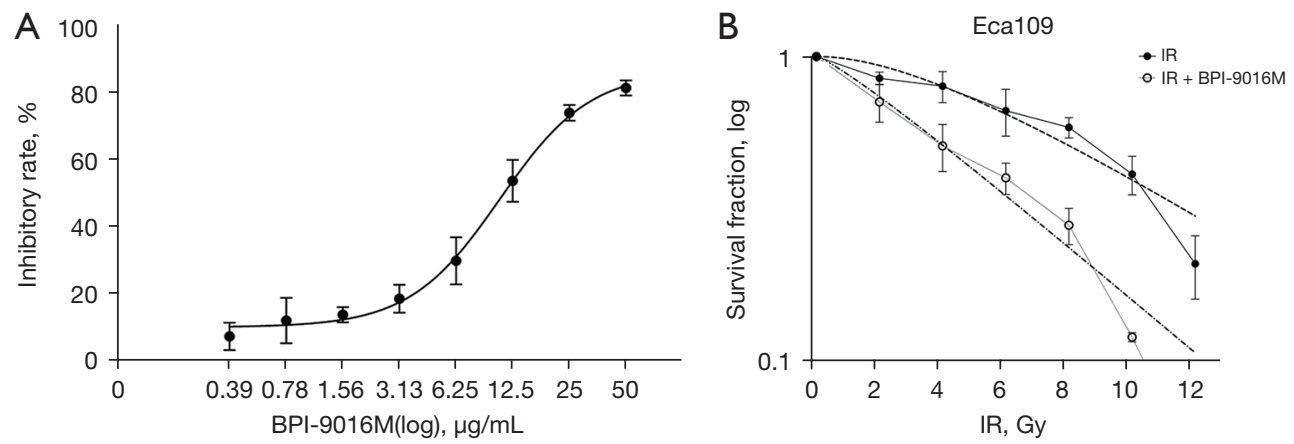

Figure 1 BPI-9016M- and/or IR-inhibited Eca109 cells. (A) BPI-9016M inhibited the proliferation of Eca109 cells in a concentrationdependent manner. (B) Clonogenic survival assays of cells treated with IR alone compared to those treated with IR and BPI-9016M. IR, irradiation.

Table 1 Effect of BPI-9016M on the radiobiological parameters of Eca109 cells

\begin{tabular}{lccccc}
\hline Group & $\mathrm{N}$ & $\mathrm{Dq}$ & $\mathrm{D} 0$ & $\mathrm{SF} 2$ & SER \\
\hline $\mathrm{IR}$ & 2.044 & 4.68 & 6.553 & 0.85 & - \\
$\mathrm{IR}+\mathrm{BPI-9016M}$ & 1.217 & 1.25 & 4.953 & $0.71^{*}$ & 1.32
\end{tabular}

Calculated using the single-hit multitarget model. ${ }^{*} \mathrm{P}<0.05$ compared to IR alone. $\mathrm{N}$, extrapolation number; $\mathrm{Dq}$, quasi-field dosage; D0, average lethal dosage; SF2, survival fraction (2 Gy); SER, sensitization enhancement ratio; IR, irradiation.

\section{Immunofluorescent staining}

Immunofluorescence (IF) was performed with a FITC Immunofluorescence Detection Kit (Cell Signaling Technology, Danvers, MA, USA). Images were captured using a confocal laser scanning microscope (CLSM; FV1200; Olympus, Tokyo, Japan).

\section{TUNEL assay}

According to the manufacturer's instructions, we used In Situ Cell Death Detection Kit-POD and In Situ Cell Death Detection Kit-Fluorescein (Roche Molecular Biology, Mannheim, Germany) to quantify TUNEL-positive cells of the xenograft tumor tissues through light and fluorescence microscopy, respectively. The apoptosis percentage was considered to be the ratio of the number of TUNELpositive cells to the total number of nuclei (4 fields of view per 1,000 tumor cells) and the number of nuclei in 1 drop of PBS by CLSM at $488 \mathrm{~nm}$ excitation.

\section{Statistical analysis}

All data are presented as the mean \pm SD of three experiments. Differences between groups were determined using one-factor analysis of variance using SPSS 21.0 software (IBM Corp., Armonk, NY, USA) or the unpaired Student's $t$-test. Differences were considered statistically significant with $\mathrm{P}$ value $<0.05$.

\section{Results}

\section{BPI-9016M inbibited cell proliferation and had a radiosensitizing effect in Eca109 cells}

Eca109 cells were exposed to different concentrations (0.391, $0.781,1.563,3.125,6.250,12.50,25$, and $50 \mu \mathrm{g} / \mathrm{mL}$ ) of BPI$9016 \mathrm{M}$ for 48 hours. BPI-9016M reduced the viability of Eca109 cells in a concentration-dependent manner (Figure 1A). Cells were pretreated with $3.0 \mu \mathrm{g} / \mathrm{mL}$ BPI-9016M for 24 hours and then irradiated at $0,2,4,6,8,10$, or 12 Gy. Surviving colonies were counted and plotted using a multitarget click mathematical model (Table 1 and Figure $1 B)$. The SER value was 1.32 . Compared IR alone, BPI9016M decreased the average lethal dosage of IR, indicating BPI-9016M reduced the ability of sublethally injured cells to repair radiation-induced damage.

\section{BPI-9016M did not significantly affect cell cycle distribution in irradiated Eca109 cells}

Flow cytometry was performed to explore the effect of the c-Met inhibitor on cell cycle distribution in irradiated Eca109 

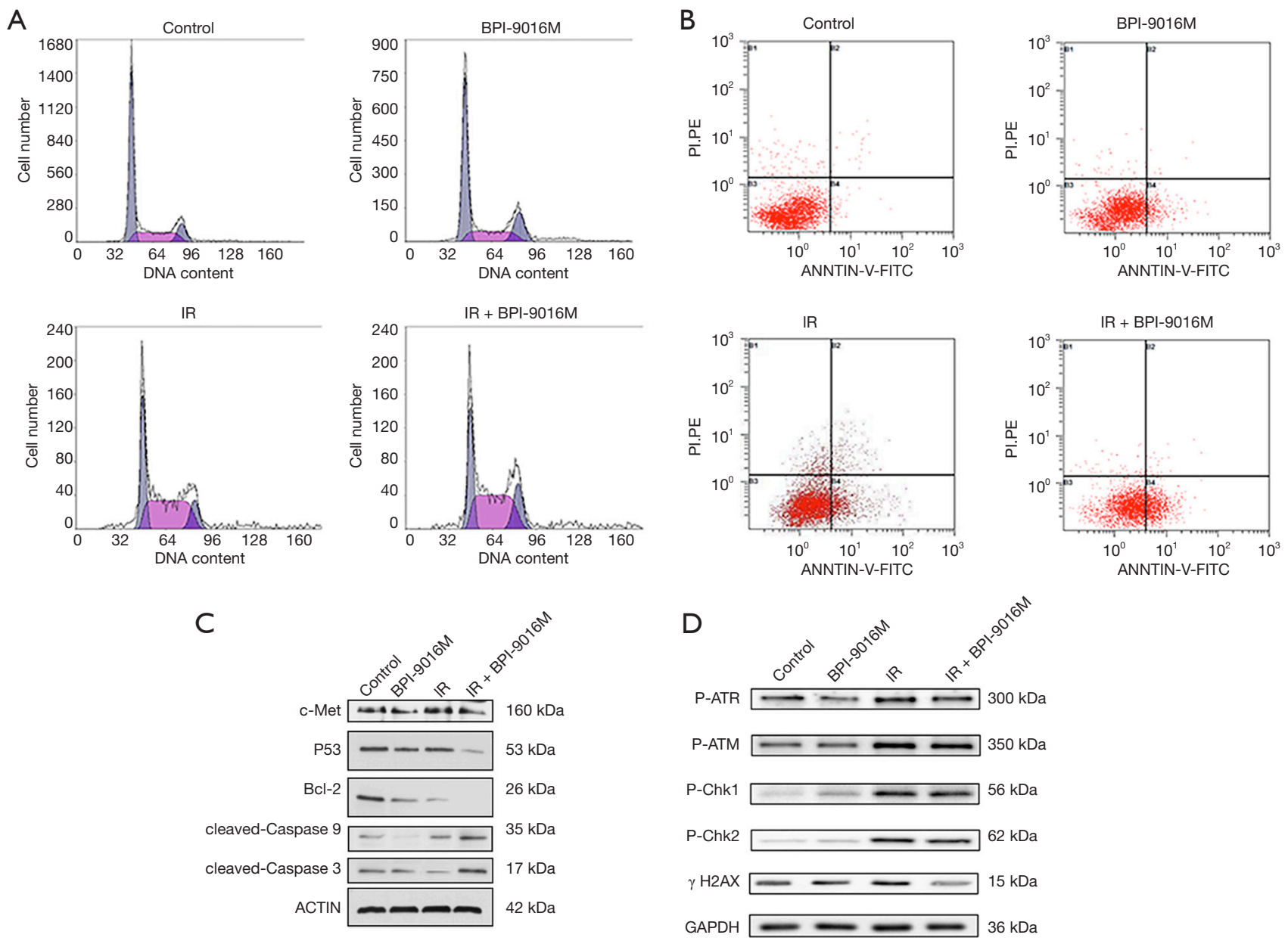

Figure 2 BPI-9016M slightly affected cell distribution but enhanced apoptosis and inhibited DNA damage repair pathway in irradiated Eca109 cells. (A) BPI-9016M slightly increased the accumulation of S phase cells with a concomitant rise in the G2/M fraction. (B) Flow cytometry analysis of apoptotic cells after Annexin V/PI double staining. (C) Representative western blot analysis of apoptosisrelated proteins. (D) Representative western blot analysis of homologous recombination repair-related proteins. PI, propidium iodide; IR, irradiation; PE, phycoerythrin; FITC, fluorescein isothiocyanate; GAPDH, glyceraldehyde-3-phosphate dehydrogenase (was used as a loading control).

cells. BPI-9016M (3.0 $\mu \mathrm{g} / \mathrm{mL}, 48$ hours) slightly increased the accumulation of $\mathrm{S}$ phase cells with a concomitant rise in the G2/M fraction (Figure $2 A$ and Table 2). However, these changes were not significant.

\section{BPI-9016M significantly enhanced apoptosis in irradiated Eca109 cells}

Annexin V-FITC and PI dual staining revealed that 6 Gy IR plus BPI-9016M $(3.0 \mu \mathrm{g} / \mathrm{mL})$ synergistically induced apoptosis (Figure 2B). The differences between the combined treatment and single treatments were statistically significant $(\mathrm{P}<0.05$; Table 2$)$.

Western blotting revealed lower levels of c-Met, mutant P53, and Bcl-2, and higher levels of cleaved caspase 3 and cleaved caspase 9 in cells treated with 6 Gy IR plus BPI$9016 \mathrm{M}$ compared to cells treated with BPI-9016M or IR alone (Figure 2C), confirming that BPI-9016M had a radiosensitizing effect by enhancing radiation-induced apoptosis in Eca109 cells. 
Table 2 Effect of BPI-9016M on cell cycle distribution and apoptosis in irradiated Eca109 cells

\begin{tabular}{lcccc}
\hline Group & G0/G1 & S & G2/M & Apoptosis \\
\hline Control & $66.2 \pm 2.7$ & $27.6 \pm 2.2$ & $6.2 \pm 1.1$ & $3.3 \pm 0.9$ \\
BPI-9016M & $67.2 \pm 2.4$ & $24.1 \pm 3.0$ & $8.7 \pm 2.8$ & $6.1 \pm 0.8$ \\
IR & $54.9 \pm 1.5$ & $36.8 \pm 2.4$ & $8.3 \pm 1.9$ & $14.5 \pm 2.6^{*}$ \\
BPI-9016M + IR & $49.6 \pm 2.3$ & $41.3 \pm 2.2^{*}$ & $9.1 \pm 1.4$ & $28.3 \pm 4.4^{\star *}$ \\
\hline
\end{tabular}

${ }^{*} \mathrm{P}<0.05$ compared to control cells; ${ }^{* *} \mathrm{P}<0.05$ compared to IR alone. IR, irradiation.
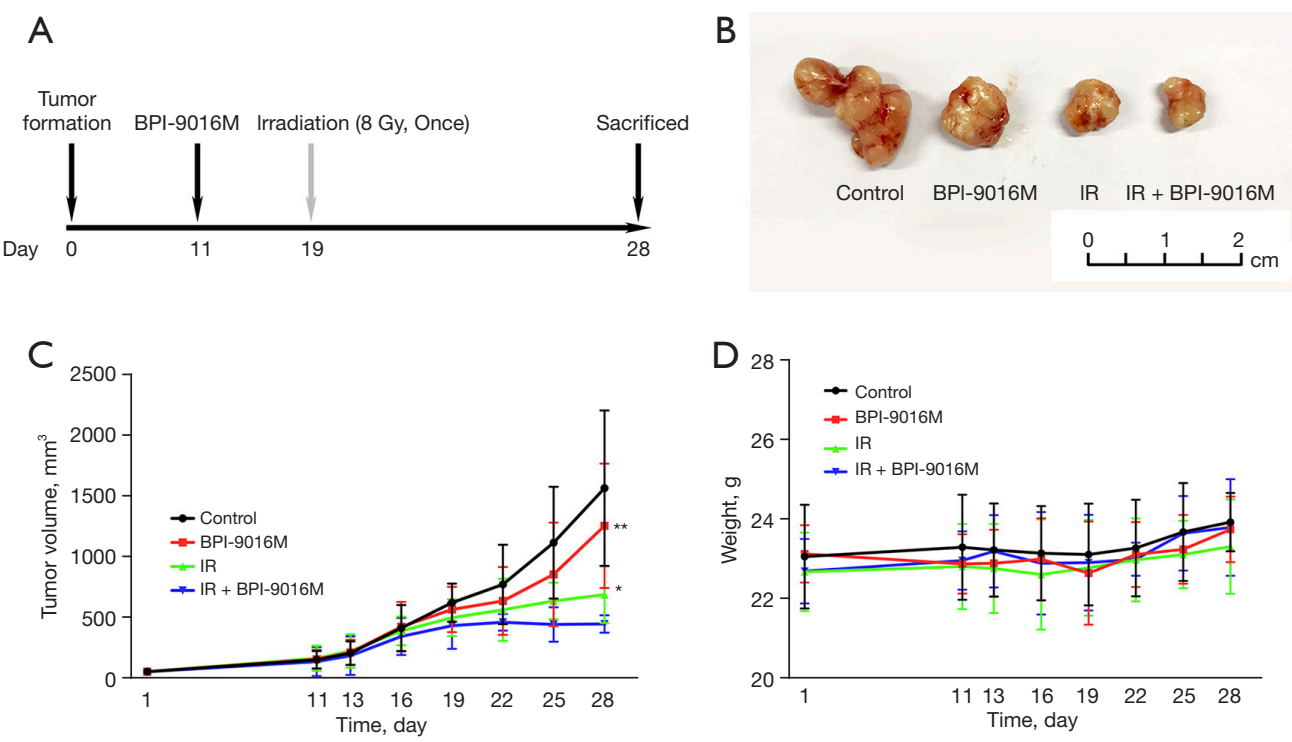

Figure 3 BPI-9016M increased the radiosensitivity of Eca109 xenograft tumors. (A) Illustration of the treatment protocol. (B) Representative images of excised tumor xenografts. (C) The growth curve of each group. (D) The body weight of each group. Data are presented as mean $\pm \mathrm{SD}$ of the 6 mice per group. ${ }^{*} \mathrm{P}<0.05$ for BPI-9016M + IR versus IR; ${ }^{* *} \mathrm{P}<0.001$ for BPI-9016M + IR versus BPI9016M. IR, irradiation.

\section{BPI-9016M inbibited the ATM- and ATR-dependent DNA damage homologous recombination repair patbways in irradiated RCA109 cells}

Western blotting also revealed IR activated the ATM/Chk2 and ATR/Chk1 pathways, as indicated by increased levels of phosphorylated ATM, Chk2, ATR, and Chk1. The H2AX histone variant was also phosphorylated in response to IR, suggesting proteins capable of stabilizing the replication fork and repairing DNA DSBs were rapidly recruited to damaged replication sites. However, the levels of phosphorylated ATM, Chk2, ATR, and Chk1 were lower in cells exposed to IR and treated with BPI-9016M than were cells exposed to radiation alone, while the level of $\mathrm{H} 2 \mathrm{AX}$ phosphorylation was significantly reduced (Figure 2D).
Taken together, these findings indicate that BPI-9016M inhibits the homologous recombination repair pathways in irritated cells, likely by inhibiting activation of the ATM/ Chk2 and ATR/Chk1 pathways in response to radiation.

\section{BPI-9016M radiosensitized Eca109 xenograft tumors}

The treatment protocol was shown in Figure 3A. Figure 3B shows images of representative Eca109 xenograft tumors. BPI-9016M combined with IR significantly delayed tumor growth compared with that observed with BPI-9016M $(\mathrm{P}<0.05)$ or IR alone $(\mathrm{P}<0.001 ;$ Figure $3 C)$. Notably, the combination therapy significantly prolonged the time it took for the tumor volume to double compared to radiation alone. However, complete tumor regression was not 
Table 3 Radiosensitizing effect of BPI-9016M in Eca109 xenograft tumors

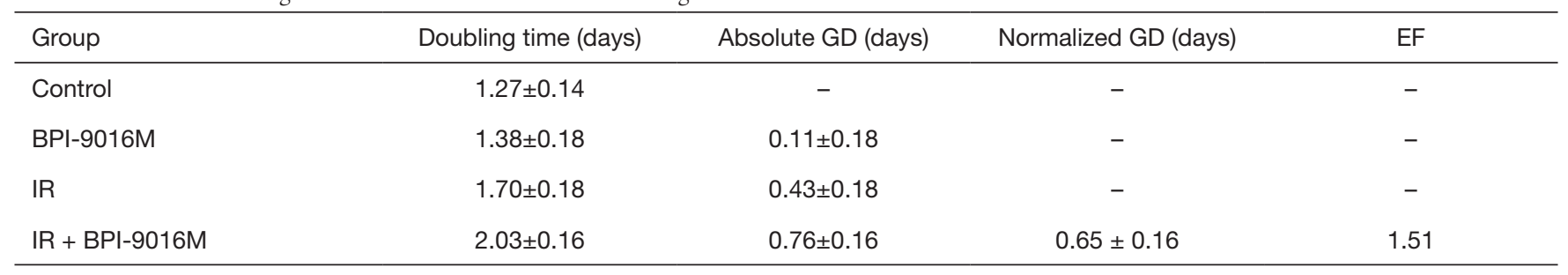

Data are based on tumor volume on day 28. Absolute GD was calculated by subtracting the doubling time of the treatment groups from that of the control group. Normalized GD was calculated by dividing the absolute GD of the IR plus BPI-9016M group from that of the $\mathrm{BPI}-9016 \mathrm{M}$ group. GD, growth delay; EF, enhancement factor; IR, irradiation.

observed for any treatment. The EF for BPI-9016M was 1.51 (Table 3). All treatments were well-tolerated with no signs of systemic toxicity, such as weight loss (Figure 3D).

\section{BPI-9016M enhanced caspase-associated apoptosis in irradiated Eca109 xenograft tumors}

IHC revealed cleaved caspase 3 was located in the membrane and cytoplasm of the tumor sections. Quantification demonstrated that the expression of cleaved caspase 3 was higher in the IR plus BPI-9016M tumors than in tumors treated with BPI-9016M or IR alone although these differences were not significant $(\mathrm{P}=0.1693$ and $\mathrm{P}=0.6171$, respectively; Figure $4 A$ ).

Based on the IF and TUNEL assay, the mean apoptotic index was $23.3 \%$ for control tumors, $44.2 \%$ for tumors treated with BPI-9016M, 40.8\% for tumors treated with IR, and $62.5 \%$ for tumors treated with IR plus BPI-9016M. The difference between the IR plus BPI-9016M tumors and IR alone tumors was significantly different $(\mathrm{P}=0.0458$; Figure $4 A)$.

\section{BPI-9016M enhanced radiation-induced DNA damage repair in irradiated Eca109 xenograft tumors}

As shown in Figure 4B, the levels of phosphorylated DNA damage repair proteins including ATM, ATR, Chk1, Chk2, and $\mathrm{H} 2 \mathrm{AX}$ were obviously increased in irradiated tumors compared to control tumors. However, consistent with the in vitro experiments, lower levels of these phosphorylated proteins were detected in both the BPI-9016M tumors and IR plus BPI-9016M tumors compared to irradiated tumors.

\section{Discussion}

This study demonstrates that the inhibition of c-Met using BPI-9016M exerts a radiosensitizing effect associated with increased radiation-induced apoptosis and attenuation of DNA damage repair mediated by homologousrecombination in ESCC cells and xenograft tumors.

Due to the effects on malignant tumor growth, invasion and metastasis, c-Met has attracted considerable interest as a potential target. Literatures indicated that the protein of overexpression and gene dysregulation of c-Met were closely related to poor prognosis and survival of patients with malignant carcinoma including $\operatorname{ESCC}(10,23,24)$. A previous study showed that high levels of HGF/c-Met in ESCC resist radiation by activating downstream PI3K/ Akt axis, regulating DNA damage repair, inducing G2/M checkpoints and down-regulating the apoptotic signaling pathway (11).

The resistance of both ionizing radiation and DNAdamaging chemotherapeutic agents was previously found to have an impact on the activation of c-Met signaling (25). Fan et al. demonstrated that DNA fragmentation caused by DNA-damaging agents can be decreased by pretreating breast cancer cells with HGF (26) and subsequently showed this effect to be mediated by c-Met via the PI3K/AKT pathway (27). Our research revealed that the ATR-Chk1/ ATM-Chk2 pathways represent an alternative mechanism of radiosensitization via a c-Met inhibitor in ESCC. These data are in agreement with previous reports demonstrating that inhibition of c-Met could reduce ATR and Chk1 phosphorylation level in gastric carcinoma cells (28). ATR and Chk1 are considered to be active at low levels, even in the calm cell cycles and especially in S phase (29). The persistence of unrepaired DNA DSBs during $S$ phase and apoptosis has been associated with the lack of homologous recombination repair (30,31); these finding may explain why BPI-9016M slightly improves the accumulation of S phase cells when combined with IR. Radiation and genotoxins potently activate ATM and Chk2 that induce DSBs, but only weakly, which is mostly likely due to drugs preventing DNA 

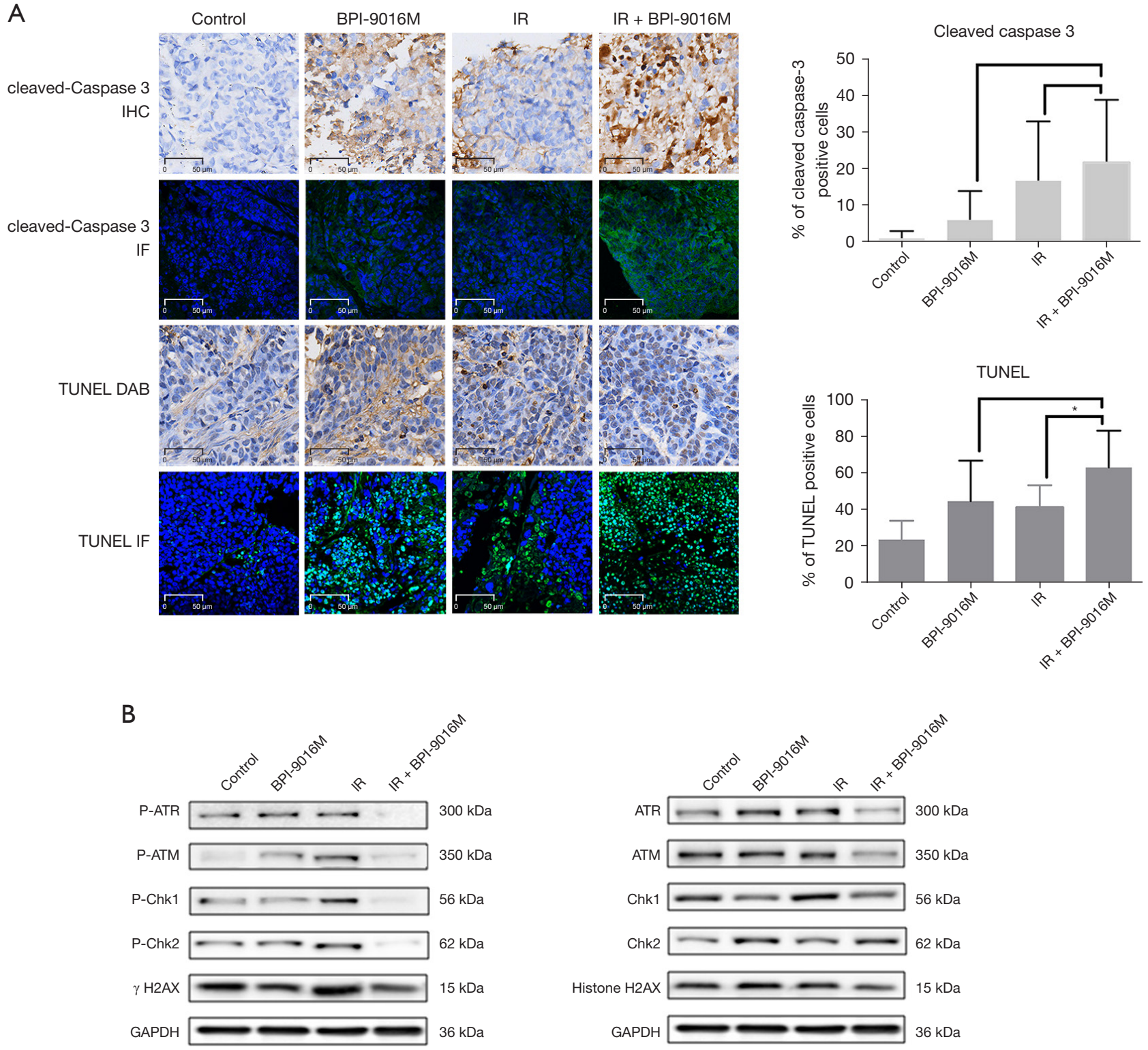

Figure 4 BPI-9016M enhanced apoptosis and inhibited radiation-induced DNA damage repair in irradiated Eca109 xenograft tumors. (A) Representative light microscope images of cleaved caspase 3 immunohistochemistry and laser scanning confocal microscopy images of immunofluorescent TUNEL staining (both shown in green) in tumor xenograft sections. ${ }^{*} \mathrm{P}<0.05$ (IR + BPI-9016M compared to IR alone). (B) Western blot analysis of homologous recombination repair-related proteins in xenograft tumor tissues. TUNEL, terminal deoxynucleotidyl transferase dUTP nick end labeling; IR, irradiation; IHC, immunohistochemical; IF, immunofluorescence; GAPDH, glyceraldehyde-3-phosphate dehydrogenase (was used as a loading control).

replication without causing damage (32). In undamaged cells, ATM is considered to be inactive homodimer, which is rapidly autophosphorylated in response to DSBs. This phenomenon also leads to its dissociation to partially active monomers (33). Once activated, Chk2 is considered to be isolated from the injured site and dispersed throughout the nucleus in the form of monomers, acting on multiple substrates (e.g., tumor suppressor P53) involved in cell cycle progression, apoptosis, and gene transcription (30,33,34). Through interacting with the MRE11/RAD50/ 
NBS1 complex (MRN) sensor complex, ATM monomers are recruited to DSBs (33). ATMs are activated and act locally on multiple substrates at sites of damage. Local substrates include the variant histone $\mathrm{H} 2 \mathrm{AX}$ which is a component of the $\gamma$-H2AX histone marker associated with DNA damage (35). We found that the level of phosphorylated H2AX was obviously increased by IR, while $\mathrm{H} 2 \mathrm{AX}$ phosphorylation was inhibited in cells which were exposed to BPI-9016M plus IR. This phenomenon indicated that BPI-9016M plus IR can cause aberrant $\mathrm{H} 2 \mathrm{AX}$ function. However, in the further studies, other cells lines with known $\mathrm{H} 2 \mathrm{AX}$ status should be used to evaluate the influence of $\mathrm{H} 2 \mathrm{AX}$ on the c-Met-mediated abrogation of radiosensitivity that is regulated by the ATM-Chk2 signal pathway.

Caspases are essential mediators which play an important role in both mitochondrial-initiated and death receptor-initiated apoptosis. Specific cleavage of numerous key cellular proteins is catalyzed by death protease caspase 3 (36). Compared to IR alone, BPI-9016M combined with IR significantly improved the levels of cleaved caspase 3 and cleaved caspase 9 in Eca109 cells. The Bcl-2 family, which comprises proapoptotic (including Bax and Bak) and anti-apoptotic (including Bcl-2 and Bcl-xl) members, is the main medium of apoptosis (36). A high Bcl-2:Bax ratio is considered to play an important role in apoptosis (37). We discovered IR caused the expression of Bcl-2, whereas BPI-9016M combined with IR significantly downregulated the expression of Bcl-2.

The multitarget click mathematical model formula (38) revealed that BPI-9016M decreased the D0, Dq, and extrapolation number $(\mathrm{N})$ values of irradiated Eca109 cells, and enhanced radiosensitivity in vitro, with a SER value of 1.40. The EF in the tumor xenograft model was 1.51.

In the first clinical trial of radiation and c-Met inhibition performed, the phase I safety portion demonstrated that patients with newly diagnosed high-grade glioma could generally tolerate administration of cabozantinib at $40 \mathrm{mg}$ daily with radiation plus temozolomide and postradiation temozolomide, with cabozantinib not showing pharmacokinetic interactions with concurrent temozolomide (39). In China, a safety trial for BPI$9016 \mathrm{M}$ in patients with advanced solid tumors is under way (NCT02478866) (40). Given the encouraging preclinical and clinical data, it is logical to further explore whether inhibition of c-Met signaling can enhance the effects of radiation therapy in ESCC.

The study has several limitations. First, additional esophageal cancer cell lines should be tested to confirm the radiosensitizing effects of BPI-9016M. Second, we need to confirm if suppression of c-Met downstream signaling results in the same enhancement of radiosensitivity as that of BPI9016M. Third, BPI-9016M is a multi-target inhibits that simultaneously inhibit c-Met and AXL, and article showed that inhibition of AXL in TP53-deficient non-small cell lung cancer cell lines increased DNA damage and induced replication stress, further experiments are necessary to evaluate the influence of BPI-9016M on the AXL signaling pathway in Eca109 cells. Finally, we are not yet clear whether the c-MET inhibitor BPI-9016M also enhances the radiosensitivity of the ESCC cell line Eca109 by inhibiting the nonhomologous end joining repair (NHEJ) pathway.

In conclusion, the c-Met inhibitor BPI-9016M can potently radiosensitize the ESCC cell line Eca109. BPI$9016 \mathrm{M}$ appears to inhibit the ATR-Chk2/ATM-Chk1 pathways to enhance tumor cell killing through both colnventional pathways (for example, increasing apoptosis), but also by triggering novel mechanisms, such as the homologour recombination repair pathway that prevents activation of ATM- and ATR-dependent DNA damage. Clearly, the next challenge is to determine whether the encouraging effects of novel c-Met inhibitors in preclinical models can be replicated in the clinic.

\section{Acknowledgments}

Funding: None.

\section{Footnote}

Reporting Checklist: The authors have completed the ARRIVE reporting checklist. Available at https://dx.doi. org/10.21037/atm-21-6586

Data Sharing Statement: Available at https://dx.doi. org/10.21037/atm-21-6586

Conflicts of Interest: All authors have completed the ICMJE uniform disclosure form (available at https://dx.doi. org/10.21037/atm-21-6586). The authors have no conflicts of interest to declare.

Ethical Statement: The authors are accountable for all aspects of the work in ensuring that questions related to the accuracy or integrity of any part of the work are appropriately investigated and resolved. Animal treatments were performed according to institution-approved 
protocols. The animal experiments were approved by the Institutional Animal Care and Use Committee of Zhejiang Cancer Research Institute (Hangzhou, Zhejiang, China) (No. 2021-12-005), in compliance with the National Institutes of Health Guide for the Care and Use of Laboratory Animals.

Open Access Statement: This is an Open Access article distributed in accordance with the Creative Commons Attribution-NonCommercial-NoDerivs 4.0 International License (CC BY-NC-ND 4.0), which permits the noncommercial replication and distribution of the article with the strict proviso that no changes or edits are made and the original work is properly cited (including links to both the formal publication through the relevant DOI and the license). See: https://creativecommons.org/licenses/by-nc-nd/4.0/.

\section{References}

1. Guo M, Zhao YD, Yang HJ, et al. Analysis of clinicopathological characteristics for 5406 cases of esophageal neoplasm. Chinese Journal of Cancer Prevention and Treatment 2008;15:54-6.

2. Zhao K, Wang C, Shi F, et al. Lymphocyte-monocyte ratio as a predictive marker for pathological complete response to neoadjuvant therapy in esophageal squamous cell carcinoma. Transl Cancer Res 2020;9:3842-53.

3. Hatogai K, Fujii S, Kojima T, et al. Large-scale comprehensive immunohistochemical biomarker analyses in esophageal squamous cell carcinoma. J Cancer Res Clin Oncol 2017;143:2351-61.

4. Lee MS, Mamon HJ, Hong TS, et al. Preoperative cetuximab, irinotecan, cisplatin, and radiation therapy for patients with locally advanced esophageal cancer. Oncologist 2013;18:281-7.

5. Mesteri I, Schoppmann SF, Preusser M, et al. Overexpression of CMET is associated with signal transducer and activator of transcription 3 activation and diminished prognosis in oesophageal adenocarcinoma but not in squamous cell carcinoma. Eur J Cancer 2014;50:1354-60.

6. Boland PM, Meyer JE, Berger AC, et al. Induction Therapy for Locally Advanced, Resectable Esophagogastric Cancer: A Phase I Trial of Vandetanib (ZD6474), Paclitaxel, Carboplatin, 5-Fluorouracil, and Radiotherapy Followed by Resection. Am J Clin Oncol 2017;40:393-8.

7. Suntharalingam M, Winter K, Ilson D, et al. Effect of the Addition of Cetuximab to Paclitaxel, Cisplatin, and Radiation Therapy for Patients With Esophageal Cancer: The NRG Oncology RTOG 0436 Phase 3 Randomized Clinical Trial. JAMA Oncol 2017;3:1520-8.

8. Han Y, Luo Y, Zhao J, et al. Overexpression of c-Met increases the tumor invasion of human prostate $\mathrm{LNCaP}$ cancer cells in vitro and in vivo. Oncol Lett 2014; 8:1618-24.

9. Abboud HS, Camuzi D, Rapozo DC, et al. MET overexpression and intratumor heterogeneity in esophageal squamous cell carcinoma. Braz J Med Biol Res 2021;54:e10877.

10. Xu Y, Peng Z, Li Z, et al. Expression and clinical significance of c-Met in advanced esophageal squamous cell carcinoma. BMC Cancer 2015;15:6.

11. Chen GZ, Dai WS, Zhu HC, et al. Foretinib Enhances the Radiosensitivity in Esophageal Squamous Cell Carcinoma by Inhibiting Phosphorylation of c-Met. J Cancer 2017;8:983-92.

12. De Bacco F, Luraghi P, Medico E, et al. Induction of MET by ionizing radiation and its role in radioresistance and invasive growth of cancer. J Natl Cancer Inst 2011;103:645-61.

13. Cui X, Zheng X, Jiang J, et al. Simultaneous determination of a novel c-Met/AXL dual-target small-molecule inhibitor BPI-9016M and its metabolites in human plasma by liquid chromatography-tandem mass spectrometry: Application in a pharmacokinetic study in Chinese advanced solid tumor patients. J Chromatogr B Analyt Technol Biomed Life Sci 2017;1068-1069:33-40.

14. Hu X, Zheng X, Yang S, et al. First-in-human phase I study of BPI-9016M, a dual MET/Axl inhibitor, in patients with non-small cell lung cancer. J Hematol Oncol 2020;13:6.

15. Watson GA, Zhang X, Stang MT, et al. Inhibition of c-Met as a therapeutic strategy for esophageal adenocarcinoma. Neoplasia 2006;8:949-55.

16. Jhawer M, Kindler HL, Wainberg Z, et al. Assessment of two dosing schedules of GSK1363089 (GSK089), a dual MET/VEGFR2 inhibitor, in metastatic gastric cancer (GC): Interim results of a multicenter phase II study. J Clin Oncol 2009;27:abstr 4502.

17. Cunningham D, Tebbutt NC, Davidenko I, et al. Phase III, randomized, double-blind, multicenter, placebo $(\mathrm{P})$ controlled trial of rilotumumab (R) plus epirubicin, cisplatin and capecitabine (ECX) as first-line therapy in patients (pts) with advanced MET-positive (pos) gastric or gastroesophageal junction (G/GEJ) cancer: RILOMET-1 study. J Clin Oncol 2015;33:abstr 4000. 
18. Kwak EL, LoRusso P, Hamid O, et al. Clinical activity of AMG 337, an oral MET kinase inhibitor, in adult patients (pts) with MET-amplified gastroesophageal junction (GEJ), gastric (G), or esophageal (E) cancer. J Clin Oncol 2015;33:abstr 1 .

19. Clark JD, Gebhart GF, Gonder JC, et al. Special Report: The 1996 Guide for the Care and Use of Laboratory Animals. ILAR J 1997;38:41-8.

20. Qin Q, Cheng H, Lu J, et al. Small-molecule survivin inhibitor YM155 enhances radiosensitization in esophageal squamous cell carcinoma by the abrogation of G2 checkpoint and suppression of homologous recombination repair. J Hematol Oncol 2014;7:62.

21. Sun Z, Liu Q, Ye D, et al. Role of c-Met in the progression of human oral squamous cell carcinoma and its potential as a therapeutic target. Oncol Rep 2018;39:209-16.

22. Lin G, Sun XJ, Han QB, et al. Epidermal growth factor receptor protein overexpression and gene amplification are associated with aggressive biological behaviors of esophageal squamous cell carcinoma. Oncol Lett 2015;10:901-6.

23. Ren JL, Wu HF, Wang WJ, et al. C-Met as a potential novel prognostic marker in squamous cell carcinoma and adenocarcinoma of esophagus: evidence from a metaanalysis. Panminerva Med 2017;59:97-106.

24. Awad MM, Oxnard GR, Jackman DM, et al. MET Exon 14 Mutations in Non-Small-Cell Lung Cancer Are Associated With Advanced Age and Stage-Dependent MET Genomic Amplification and c-Met Overexpression. J Clin Oncol 2016;34:721-30.

25. Bhardwaj V, Cascone T, Cortez MA, et al. Modulation of c-Met signaling and cellular sensitivity to radiation. Cancer 2013;119:1768-75.

26. Fan S, Wang JA, Yuan RQ, et al. Scatter factor protects epithelial and carcinoma cells against apoptosis induced by DNA-damaging agents. Oncogene 1998;17:131-41.

27. Giordano S, Columbano A. Met as a therapeutic target in HCC: facts and hopes. J Hepatol 2014;60:442-52.

28. Medová M, Aebersold DM, Blank-Liss W, et al. MET Inhibition Results in DNA Breaks and Synergistically Sensitizes Tumor Cells to DNA-Damaging Agents Potentially by Breaching a Damage-Induced Checkpoint Arrest. Genes Cancer 2010;1:1053-62.

29. Syljuåsen RG, Sørensen CS, Hansen LT, et al. Inhibition of human Chk1 causes increased initiation of DNA replication, phosphorylation of ATR targets, and DNA breakage. Mol Cell Biol 2005;25:3553-62.

30. Myers SH, Ortega JA, Cavalli A. Synthetic Lethality through the Lens of Medicinal Chemistry. J Med Chem 2020;63:14151-83.

31. Romano M, Frapolli R, Zangarini M, et al. Comparison of in vitro and in vivo biological effects of trabectedin, lurbinectedin (PM01183) and Zalypsis® (PM00104). Int J Cancer 2013;133:2024-33.

32. Ramkumar K, Stewart CA, Cargill KR, et al. AXL Inhibition Induces DNA Damage and Replication Stress in Non-Small Cell Lung Cancer Cells and Promotes Sensitivity to ATR Inhibitors. Mol Cancer Res 2021;19:485-97.

33. Buchanan IM, Scott T, Tandle AT, et al. Radiosensitization of glioma cells by modulation of Met signalling with the hepatocyte growth factor neutralizing antibody, AMG102. J Cell Mol Med 2011;15:1999-2006.

34. Eren MK, Kartal NB, Pilevneli H. Oncogenic WIP1 phosphatase attenuates the DNA damage response and sensitizes p53 mutant Jurkat cells to apoptosis. Oncol Lett 2021;21:479.

35. Barroso SI, Aguilera A. Detection of DNA Double-Strand Breaks by $\gamma$-H2AX Immunodetection. Methods Mol Biol 2021;2153:1-8.

36. Siddiqui WA, Ahad A, Ahsan H. The mystery of BCL2 family: Bcl-2 proteins and apoptosis: an update. Arch Toxicol 2015;89:289-317.

37. Sharifi S, Barar J, Hejazi MS, et al. Roles of the Bcl-2/Bax ratio, caspase- 8 and 9 in resistance of breast cancer cells to paclitaxel. Asian Pac J Cancer Prev 2014;15:8617-22.

38. Gardner SN. A mechanistic, predictive model of dose-response curves for cell cycle phase-specific and -nonspecific drugs. Cancer Res 2000;60:1417-25.

39. Schiff D, Desjardins A, Cloughesy T, et al. Phase 1 dose escalation trial of the safety and pharmacokinetics of cabozantinib concurrent with temozolomide and radiotherapy or temozolomide after radiotherapy in newly diagnosed patients with high-grade gliomas. Cancer 2016;122:582-7.

40. Shi Y. Safety and Pharmacokinetics of BPI-9016M in Patients With Advanced Solid Tumors. 2015. Available online: https://clinicaltrials.gov/ct2/show/NCT02478866 (Accessed 21 May, 2015).

Cite this article as: Jiang C, Han S, Sun X, Xu Y, Feng J, Shang J. Radiosensitizing effect of c-Met kinase inhibitor BPI9016M in esophageal squamous cell carcinoma cells in vitro and in vivo. Ann Transl Med 2021;9(24):1799. doi: 10.21037/atm21-6586 